\title{
Model for determining the optimal number of lanes in toll stations
}

\author{
Fei Zheng \\ Department of North China Electric Power University（Baoding）, Baoding 071000, China \\ 18331125631@163.com
}

Keywords: BP-Neural Networks, Queuing Theory , Goal programming

\begin{abstract}
I design a rational toll plaza layout and the congestion problem of the vehicle passing th rough the toll station is optimized reasonably.By analyzing the behavior process, the arrival distrib ution and the distribution model of the vehicles at the toll station,we get a reasonable number of con ventional lanes, toll stations

In the export confluence area, the traffic volume is large and the road is complex. Vehicles in accelerated lane will have a common merger behavior, it often causes the trunk line of traffic flowing disorder, reduces the traveling speed of traffic flow, causes the severe delay, and causes the traffic accident .So the confluence area is the accident-prone areas of the highway. Based on the research on the layout and design of toll station, we use the BP-neural network method to simulate the driver in the confluence of the highway entrance and line decision-making process. By comparing the capacity of the conventional toll plaza with the same conditions, and then comparing the results of the model forecast with the actual situation, the results are more consistent. Thus I obtain a more reasonable merging model.
\end{abstract}

\section{Introduction}

Expressway has become an important symbol of modernization. In the United States, New Jersey, traffic is large. The usage rage of highways are also more. The highway is more comfortable than the ordinary road. Now science and technology is growing, the number of vehicles gradually increased. As the purchasing power of people increases, and the development of tourism, there will be more vehicles into the highway. Self-driving vehicles are also being developed, after which there will be more self-driving vehicles into the highway. With the increase in social demand, more and more large trucks into the highway. So the traffic flow is gradually increased. From the traffic volume analysis, the highway traffic volume is close to saturation, there are some ways need to be adjusted. Confluence has not changed. It can be improved from the confluence of ways to facilitate the increase in traffic and economic development. But also it is conducive to people's comfort increase, and improve the service level of the expressway, showing the degree of modernization.

The safety of expressway is also becoming more and more serious. Expressway on the vehicle speed is very fast, which is already prone to traffic accidents. With the increasing of traffic flow, the probability of traffic accidents increases. Accidents on the highway are often very serious. Some people may lose their lives.

Toll plaza is a place to charge. There are three kinds of toll stations. At present, toll stations are fewer. The number of ETCs is increasing. ETC is a road that can be passed directly. The increase in ETC will cause an increase in traffic flow. Toll plaza design is reasonable, to a large extent, can increase the number of vehicles through. Toll plaza design should follow a lot of principles. Confluence of the program should also pay attention to security issues. According to the results of the study it has been shown that the confluence of the probability of an accident. Acceleration lane way and length also need to consider the problem. 


\section{Restatement of the problem}

"Obstacle charging" is a method of charging across the highway, the toll station is perpendicular to the direction of traffic flow. Usually toll stations is more than traffic lines .Therefore, when driving out of the toll station, the vehicle must "flood" from a larger number of toll lane exits to a smaller number of conventional lanes. When the traffic pressure is greater, the toll station export confluence has become the cause of traffic congestion and the impact of traffic efficiency is an important reason. Therefore, in order to alleviate the traffic pressure, we should be a reasonable toll station transformation. In order to make the confluence of the pressure relief, we must first control the number of vehicles into the toll plaza. To do this we have to carry out a reasonable calculation of the number of conventional road lanes, and then according to the number of lanes to determine the number of toll stations and the proportion of ETC and MTC planning.

\section{Establish the Mathematical Model}

\subsection{Determination of number of lanes}

To the highway toll plaza transformation, pre-feasibility of the project is very important. To determine the technical standards of the highway is more important, such as the number of lanes set too much, much larger than the expected traffic volume requirements, it will result in resources and financial input Of the waste; to the contrary ,it may cause the highway just opened soon need to broaden, also caused a waste. Here we briefly introduce the highway engineering feasibility study phase to determine the number of lanes and the process.

We use the design speed of $100 \mathrm{~km} / \mathrm{h}$ standard construction. According to "Highway Route Design Code" , designing speed of $100 \mathrm{~km} / \mathrm{h}$ expressway service level requirements is in following Table .

Highway service level classification

\begin{tabular}{|c|l|l|c|c|}
\hline \multirow{2}{*}{$\begin{array}{c}\text { Service level } \\
\text { classificatio } \\
\mathrm{n}\end{array}$} & \multirow{2}{*}{\begin{tabular}{c} 
density \\
\cline { 3 - 5 }
\end{tabular}} & & \multicolumn{3}{|c|}{ Design speed/km/h } \\
\cline { 3 - 5 } & & $\mathrm{V} / \mathrm{km} / \mathrm{h}$ & $\mathrm{V} / \mathrm{C}$ & $\begin{array}{l}\text { Maximum Service Traffic } \\
\text { Volume/ } p c u \cdot(h \cdot \ln )^{-1}\end{array}$ \\
\hline I & $\leq 7$ & $\geq 96$ & 0.33 & 700 \\
\hline II & $\leq 18$ & $\geq 79$ & 0.67 & 1400 \\
\hline III & $\leq 25$ & $\geq 71$ & 0.86 & 1800 \\
\hline IV & $>25$ & $<71$ & 1.0 & $<2100$ \\
\hline
\end{tabular}

In the planning and construction of the highway toll station, according to the standard delay - flow curve (American Highway Service Standard), the maximum service traffic volume at all levels of service level can be obtained by revising the service levels at all levels when the highway toll station is being planned and constructed. 


\subsubsection{Actual basic capacity}

\section{Parameter}

\begin{tabular}{|l|l|}
\hline Symbol & \multicolumn{1}{c|}{ Meaning } \\
\hline$C_{B T}$ & Actual basic traffic capacity of expressway veh/(h·ln) \\
\hline$C_{B}$ & Basic traffic capacity of expressway veh/(h·ln) \\
\hline$f_{N}$ & Lane correction factor \\
\hline$f_{H V}$ & Traffic composition correction factor. \\
\hline \multicolumn{1}{|c|}{$\boldsymbol{P}_{\boldsymbol{i}}$} & $\begin{array}{l}\text { Medium-sized vehicles, large vehicles, trailers (i) traffic volume of the } \\
\text { total traffic percentage }\end{array}$ \\
\hline $\boldsymbol{E}_{\boldsymbol{i}}$ & Medium-sized vehicles, large cars, trailers(i), vehicle conversion factor \\
\hline$f_{P}$ & The correction factor for the driver condition, $f_{P}=0.98$. \\
\hline
\end{tabular}

\section{Calculation formula}

The actual basic traffic capacity of a lane on a freeway is calculated as follows:

$$
\begin{aligned}
& C_{B T}=C_{B} X f_{N} X f_{H V} \mathrm{X} f_{P} \\
& f_{H V}=\mathbf{1} /\left[\mathbf{1}+\sum \boldsymbol{P i}\left(E_{i}-\mathbf{1}\right)\right] \\
& f_{H V}=\mathbf{0 . 7 5 3} \\
& C_{B T}=\mathbf{2} 100 \times \mathbf{0 . 9 7} \times \mathbf{0 . 7 5} \times \mathbf{0 . 9 8}=\mathbf{1} 503 \mathrm{veh} /(\mathbf{h} \cdot \mathbf{l n}) 。
\end{aligned}
$$

\begin{tabular}{|c|c|}
\hline Symbol & Meaning \\
\hline$C_{B T}$ & Design capacity veh/(h·ln) \\
\hline$C_{D}$ & Actual basic traffic capacity of expressway veh/(h·ln) \\
\hline
\end{tabular}

\subsubsection{Design capacity}

\section{- Parameter}

\section{- Capacity calculation}

Highway planning and design, it is necessary to ensure a higher level of service and vehicle operating quality, to avoid traffic soon due to traffic congestion caused by inappropriate traffic, but also take into account the level of economic development and highway construction investment.

According to "Highway Route Design Code" highway should be designed according to the two service levels, so $\mathrm{V} / \mathrm{C}=0.67$

The calculated hourly capacity of freeway design is:

$$
C_{D}=1503 \times 0.67=1007 \mathrm{veh} /(\mathrm{h} \cdot \ln )
$$

\section{Calculate the number of lanes}

\section{Parameter:}

\begin{tabular}{|r|l|}
\hline Symbol & \multicolumn{1}{|c|}{ Meaning } \\
\hline $\mathrm{N}$ & Number of unidirectional lanes \\
\hline AADT & Forecast the annual average daily traffic volume \\
\hline $\mathrm{D}$ & Direction distribution coefficient, $\mathrm{D}=0.52$ \\
\hline $\mathrm{K}$ & Design hour traffic coefficient, $\mathrm{K}=0.085$ \\
\hline
\end{tabular}

\section{- Conclusion}

The number of freeway lanes is calculated as follows:

$$
\mathrm{N}=\mathrm{AADT} \times \mathrm{K} \times \mathrm{D} / C_{D}
$$

Through calculation and analysis, we get one-way three-lane for the optimal number of lanes.

After determining the optimal number of lanes, in order to solve the confluence problem after charging, we need to solve the problem of the optimal number of toll booths, so as to solve the congestion problem at the source.

- If there are not enough toll booths, the vehicle will form a queue situation.

- If the toll station is too large, the vehicle will be congested when it is merged into a narrow highway. We are on the basis of the discussion to get 3 lanes, 7 toll booths optimization method. 


\section{Conclusions}

The design of the toll station is a comprehensive project. In this model, we mainly consider the number of toll channels, the shape of the toll plaza, and the confluence of the post-paid vehicles. Finally, we give the solution to congestion problem of toll station. In our program, we did optimization in terms of traffic capacity, footprint, and security, ultimately we determine a more reasonable form of design .The following conclusions are drawn from this modeling:

Through the forecast of traffic volume, we give a detailed calculation method of the number of lanes of expressway, which lays a foundation for further study of the number of toll lanes. It is reasonable to conclude that we have three lanes in one direction.

Next we use the average number of queues in the toll collection channel as the main parameter to evaluate the capacity of the toll station. Based on the characteristics of traffic flow at highway toll station, the method of assessing toll station capacity is given, and the queuing model of optimizing the number of toll stations is given by using queuing theory. And finally identified three lanes when the seven toll channels of the design.

\section{References}

[1] Yuan. Highway confluence area vehicle simulation and evaluation research [D]. Hefei university of technology, 2004

[2]ChiJingHao, xiao-hua zhao, RongJian Xu Fei. Based on the indication unmanned driving simulator of highway toll station design research [J]. Journal of transportation information and security, 2015, (6) : 114-119.

[3] Wen-quan li, wang, glowei. Highway ramp confluence area capacity empirical model [J]. Journal of transportation engineering, 2004, (02) : 80-84

[4] Tie-zhu li wen-quan li, Zhou Ronggui Shi Xiaofa, zhao. Highway deceleration lane confluence shunt characteristics analysis [J]. Highway traffic science and technology, 2001, (4) : 89-91.

[5] LI Wen-quan,WANG Wei , ZHOU Rong-gui .Headway characteristics of lane 1 on expressway merge area[ J] .Journal of Highway and Transportation Research and Development, 2003, 20(1):114-117. (in Chinese) 